\title{
Multifunctional Top-Coats Strategy for DSA of High- $\chi$ Block Copolymers
}

\author{
Xavier Chevalier $^{1, *}$, Cindy Gomes Correia ${ }^{2}$, Gwenaelle Pound-Lana ${ }^{3}$, Philippe Bézard ${ }^{3,4}$, Matthieu \\ Sérégé $^{3}$, Camille Petit-Etienne ${ }^{3}$, Guillaume Gay ${ }^{3}$, Gilles Cunge ${ }^{3}$, Benjamin Cabannes-Boué ${ }^{2}$, Célia \\ Nicolet $^{1}$, Christophe Navarro ${ }^{1}$, Ian Cayrefourcq ${ }^{1,5}$, Marcus Müller ${ }^{6}$, Georges Hadziioannou ${ }^{2}$, Ilias \\ Iliopoulos ${ }^{7}$, Guillaume Fleury ${ }^{2}$, and Marc Zelsmann ${ }^{3}$ \\ ${ }^{1}$ ARKEMA FRANCE, GRL, Route Nationale 117, BP34 64170 Lacq, France. \\ ${ }^{2}$ Univ. Bordeaux, CNRS, Bordeaux INP, LCPO, UMR 5629, F-33600, Pessac, France. \\ ${ }^{3}$ Univ. Grenoble Alpes, CNRS, CEA/LETI Minatec, Grenoble INP, LTM, 38000 Grenoble, France. \\ ${ }^{4}$ IMEC, Kapeldreef 75, 3001 Leuven, Belgium. \\ ${ }^{5}$ I-TEN SA, 12 Chemin du Jubin, F69570, Dardilly, France. \\ ${ }^{6}$ Georg-August Universität Göttingen, Institute for Theoretical Physics, 37077 Göttingen, Germany. \\ ${ }^{7}$ Laboratoire PIMM, Arts et Métiers Institute of Technology, CNRS, Cnam, HESAM Université, 151 \\ Boulevard de l'Hôpital, 75013 Paris, France. \\ *xavier.chevalier@arkema.com
}

\begin{abstract}
A concept of patternable top-coats dedicated to directed self-assembly of high- $\chi$ block copolymers is detailed, where the design enables a crosslinking reaction triggered by thermal or photo-activation. Nanostructured BCP areas with controlled domains orientation are selected through a straightforward top-coat lithography step with unique integration pathways. Additionally, the crosslinked nature of the material enables the suppression of the BCP dewetting, while exhibiting exceptional capabilities for the construction of 3D stacks.

Keywords: Lithography, Block copolymers, Self-assembly, Cross-linkable top-coats, Top-coat patterning, Nanopatterning, Dewetting
\end{abstract}

\section{Introduction}

The directed self-assembly (DSA) of block copolymers (BCPs) is a potential method of choice to obtain regular arrays of predetermined patterns, owing to their intrinsic periodic nature, ease of processing, and nanometer-scale dimensions of features. However, reaching aggressive dimensions below $10 \mathrm{~nm}$, i.e. below the ultimate resolution of polystyrene- $b$-poly(methyl methacrylate) (PS- $b$ PMMA) system [1], necessitates the use of high- $\chi$ BCPs $(\chi$ being the Flory-Huggins segregation parameter). Such BCPs are very often constituted of blocks presenting a large difference in their intrinsic surface energy at the targeted annealing temperature, promoting the self-assembly process. These BCPs necessitate the use of top-coats (TC) materials to balance the interfacial tension between the blocks and achieve the perpendicular orientation of nanometric features [2]. In our previous works we proposed a new design of TCs able to circumvent keys challenges posed by the integration of DSA into microelectronic nodes $[3,4]$. Here we highlight the specific properties of these TCs enabling their multifunctional use for nano-manufacturing.

\section{Experimental}

\subsection{Synthesis of TC material}

The synthesis of the TC material was performed by radical polymerization in solution with AIBN as initiator [3]. All the monomers, solvent, initiator and terminating agent were used as received.

2.2. Synthesis of poly(1-1dimethylsilacyclobutane)$b$-polystyrene (PDMSB- $b$-PS BCP) 
The synthesis of the BCP material was performed as previously reported [5].

\subsection{Preparation of thin films}

$0.9 \mathrm{wt} \%$ PDMSB- $b$-PS solutions in methyl isobutyl ketone (MIBK) were spin-coated ( $2 \mathrm{krpm})$ on neutralized $3 \times 3 \mathrm{~cm}$ Si-substrate, followed by a soft-bake at $60{ }^{\circ} \mathrm{C}$ for $1 \mathrm{~min}$ to produce BCP layers with approximatively $30 \mathrm{~nm}$ thickness. The TC material dissolved into absolute ethanol at $2 \mathrm{wt} \%$, is spin-coated on the BCP thin film at $1500 \mathrm{rpm}$. The stack is baked at $90{ }^{\circ} \mathrm{C}$ for 3 minutes, and the $\mathrm{BCP}$ is self-assembled at $240{ }^{\circ} \mathrm{C}$ for 5 minutes.

\subsection{Ellipsometry}

The film thicknesses were determined using spectroscopic ellipsometry in reflection mode with a phase modulated spectroscopic ellipsometer (UVISEL, from Horiba Scientific) on the spectral range $200-800 \mathrm{~nm}$.

\subsection{Patterning}

UV lithography was carried out with a KarlSüss MJB4 equipment equipped with a mercury arc lamp having a power of $20 \mathrm{~mW} / \mathrm{cm}^{2}$ at a wavelength of $365 \mathrm{~nm}$.

Electron-beam (e-beam) lithography was performed on a JEOL6300FS equipment at an acceleration voltage of $100 \mathrm{kV}$, with a current of 500 $\mathrm{pA}$ and a dose of $80 \mu \mathrm{C} / \mathrm{cm}^{2}$.

\subsection{Microscopy}

Top-view SEM images were acquired on a Hitachi CD-SEM H-9300 microscope with $0.5 \mathrm{kV}$ electron acceleration voltage and a $6 \mu \mathrm{A}$ current. FIB-STEM cross-sections were carried out using a Helios 450s-FEI dual-beam equipment.

\section{Results and discussion}

\subsection{Macromolecular design}

The BCP used is based on the PDMSB- $b$-PS platform (Fig. 1, left) [5]. It presents a large difference in the surface free energy of its blocks, and is able to reach dimensions below $12 \mathrm{~nm}$ in period as previously demonstrated [3]. The silicon, present in the PDMSB block, enables to increase the etch performances for the final transfer step into the underlying substrate. It avoids the use of a timeconsuming sequential-infiltration-synthesis step to provide a decent etch contrast between the two blocks.

The TC material designed is based on a methacrylic terpolymer, with a statistical architecture of $\operatorname{poly}(2,2,2-$ trifluoroethylmethacrylate- $\mathrm{co}$ -

glycidilmethacrylate-co-

hydroxyethylmethacrylate) hereafter abbreviated "FGH" (Fig. 1, middle). This TC is thus entirely organic and exhibits a great etch selectivity with respect to the $\mathrm{Si}$-containing $\mathrm{BCP}$ under $\mathrm{O}_{2}$-based plasma. The FGH material presents low $M_{\mathrm{w}}(\sim 3000$ $\mathrm{g} / \mathrm{mol}$.) and polar comonomers, favoring its solubility into alcoholic solvents (pure ethanol of IPA for instance), while the BCP material is insoluble in such solvents. Its inexpensive and available constituents, readiness of synthesis through radical polymerization, and easiness of compositional tuning, make it a material of choice for DSA applications. Moreover, FGH material can also be used as grafted or cross-linked underlayer, thanks to hydroxyl and epoxy chemical functions respectively.

The FGH solution is readily spin-coated and cross-linked on top of the BCP, the interfacial
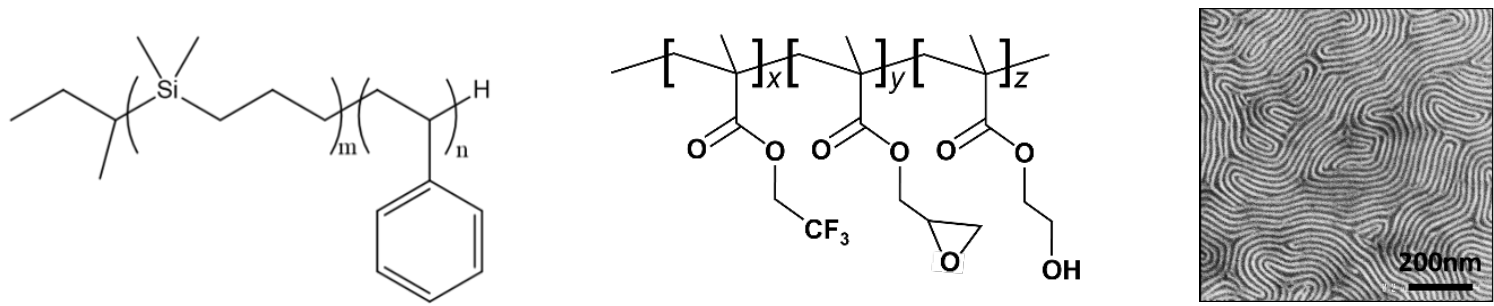

Fig. 1. Chemical structure of PDMSB- $b$-PS block copolymer (left); Structure of FGH top-coat material (middle); Top-view SEM picture showing the self-assembly of a lamellae-forming $18 \mathrm{~nm}$ period PDMSB- $b$-PS BCP obtained by using a neutral FGH material as TC and a neutralized substrate, with a $240{ }^{\circ} \mathrm{C} / 5$ minutes thermal annealing process (right). 


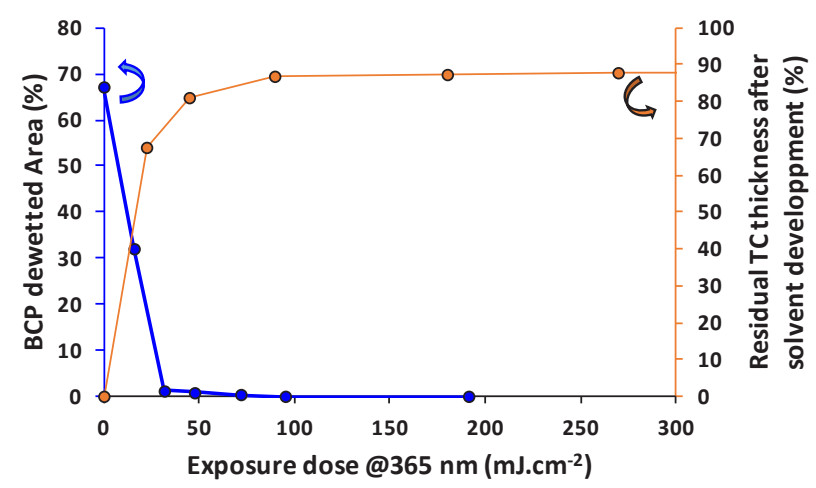

Fig. 2. Evolution of the remaining FGH thickness, after exposure to various doses of UV light $(\lambda=365 \mathrm{~nm})$ and subsequent rinse in ethanol for 2 mins (orange plot). Evolution of the dewetted area on the sample of a neutralized substrate/PDMSB$b$-PS/neutral FGH stack as a function of the UV light $(\lambda=365 \mathrm{~nm})$ exposure dose, a PEB performed at $90^{\circ} \mathrm{C}$ for 3 minutes, and subsequent thermal annealing at $260{ }^{\circ} \mathrm{C}$ for 5 minutes to promote the self-assembly of the block copolymer layer (blue plot).
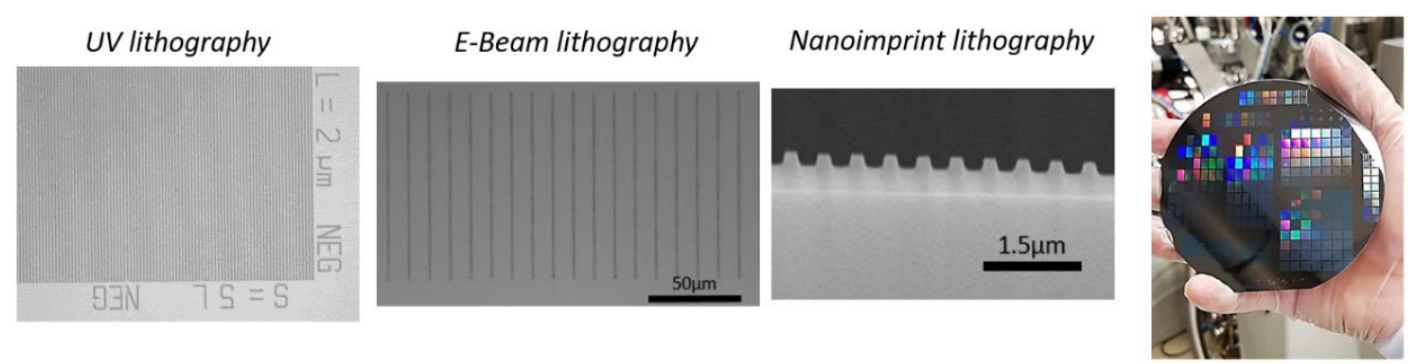

Fig. 3. Examples of FGH patterning on top of PDMSB- $b$-PS film with different techniques. A 4 inch wafer of a BCP/TC stack, where the FGH -TC material is patterned with UV light $(\lambda=365 \mathrm{~nm})$ exposure, is shown on the right.

tension of both PDMSB and PS blocks are subsequently well-balanced upon thermal annealing and, as a result, it drives the perpendicular orientation of the domains (Fig.1, right). This first functionality as "neutral" material is found for adjusted composition of FGH comonomers, selected through the synthesis.

\subsection{Cross-linking}

One of the most interesting functionalities of the FGH material is their ability to be cross-linked. Owing to the epoxy functions in FGH, the crosslinking process can be triggered either by thermal stimulus with a latent catalyst, or by photoactivation with a photoacid generator (PAG) followed by a post-exposure bake (PEB), and completed within few minutes. This particular property, and the methacrylic nature of the backbone, leads to a stiff material and confers to the FGH layer a tremendous mechanical strength. This mechanical cohesion enables to freeze the dewetting kinetic of the underlying BCP material, and thus locks the BCP film in its initially flat configuration. Although the amplification of the capillary waves responsible of the dewetting [6] is suppressed by the cross-linking of the FGH, the BCP material is still able to selfassemble into a lamellar microphase, as proven in Fig. 1, right. This unique property owned by FGH materials is illustrated in Fig. 2. It can be seen that the FGH material blended with (4phenylthiophenyl) diphenylsulfonium triflate as PAG is easily photo-cross-linked at low UV doses, even for a $365 \mathrm{~nm}$ wavelength. The FGH thickness is indeed constant for doses as low as $100 \mathrm{~mJ} / \mathrm{cm}^{2}$, followed by a 3 minutes PEB at $90{ }^{\circ} \mathrm{C}$, and then rinsed in ethanol (Fig. 2, orange plot). Meanwhile, the dewetting of the BCP film is progressively mitigated when the UV dose is increased, and is entirely suppressed for UV dose of $\sim 100 \mathrm{~mJ} / \mathrm{cm}^{2}$, even with a subsequent high temperature selfassembly annealing process as $260{ }^{\circ} \mathrm{C}$ for 5 minutes (Fig. 2, blue plot).

\subsection{Patterning}

The most appealing property of the FGH material is their ability to be patterned like a standard lithographic resist. Indeed, the chosen methacrylic platform enables the FGH materials to be patterned 


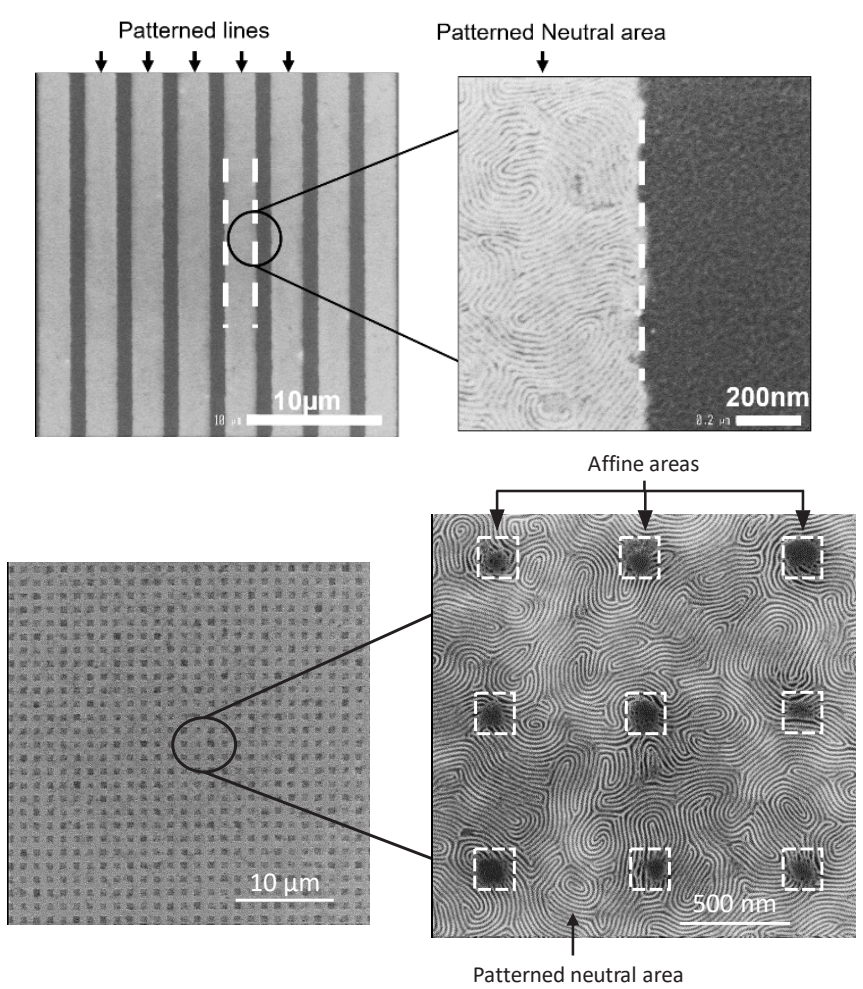

Fig. 4. Example of FGH-patterning results, demonstrating the selection of perpendicular and parallel areas of a lamellae-forming $18 \mathrm{~nm}$ period PDMSB- $b$-PS BCP with UV light $(\lambda=365 \mathrm{~nm})$ exposure (top panel) or e-beam exposure (bottom panel). The thermal annealing step was performed at $240{ }^{\circ} \mathrm{C}$ for 5 minutes.
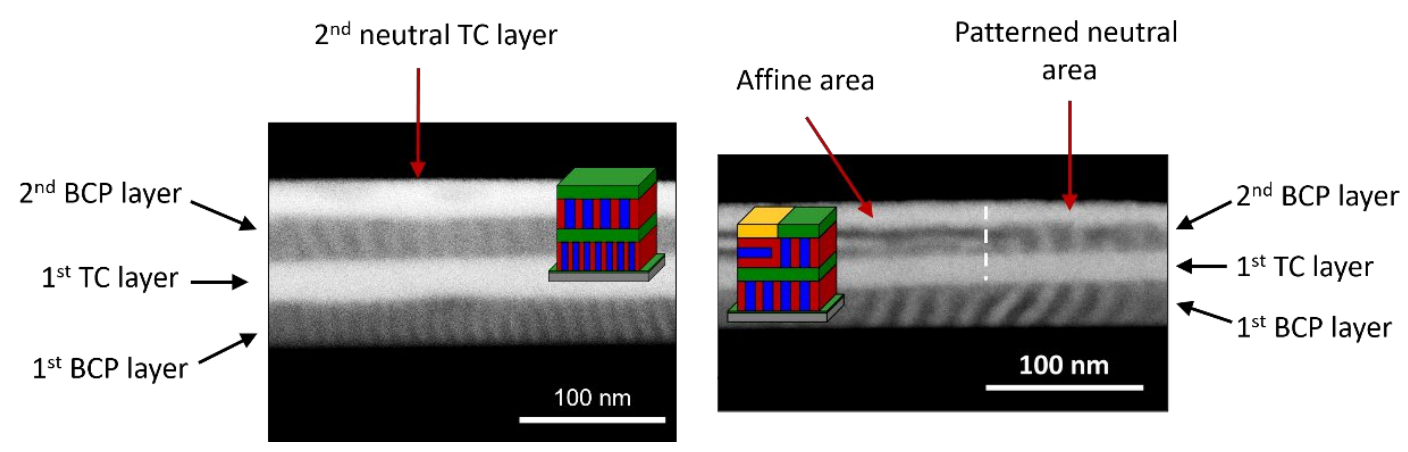

Fig. 5. Examples of 3D-multilayered PDMSB- $b$-PS film stack obtained with the specific FGH as neutral interface material, where the cross-sections are characterized with FIB-STEM technique. The first BCP layer is a lamellaeforming $12 \mathrm{~nm}$ period PDMSB- $b$-PS BCP, and the second is a lamellae-forming $18 \mathrm{~nm}$ period PDMSB- $b$-PS BCP (left). Example of a combination of BCP multilayers stack with the patterning ability of the FGH material (right). The upper patterned FGH film leads to different orientational order of the $2^{\text {nd }}$ lamellar BCP layer. A lamellae-forming 18 $n m$ period PDMSB- $b$-PS was used as first and second BCP layer.

with most of advanced lithographic techniques, as illustrated on Fig. 3 with the examples of UV $(\lambda=365 \mathrm{~nm})$, e-beam and nanoimprint techniques. In the case of UV lithography, it is worth mentioning that methacrylates are transparent down to $193 \mathrm{~nm}$ wavelength as they are the basis of many chemically amplified resist materials [7]. Moreover, the possibility of sensitization [8] even opens up a compatibility with EUV technique for advanced nodes. It is also of interest to mention that the FGH material and patterning processes can be easily scaled up to wafer sizes, as demonstrated with a $\mathrm{BCP} / \mathrm{TC}$ stack on a 4 inches wafer where the TC is patterned with UV light $(\lambda=365 \mathrm{~nm})$ on the Fig. 3, right.

The FGH material, blended with a PAG as latent catalyst, behaves as a chemically amplified negative resist, due to the nature of the cross-linking reaction through the epoxy moieties [9]. Thanks to the ease of their compositional tuning with the synthesis, the 
user is able to readily choose either the neutral or affine nature of the TC material to be patterned. This unique patterning ability of the FGH opens up new integration schemes for the DSA of block copolymers, leading to the selection of areas of interest with either perpendicular or parallel orientation of BCP domains directly through the TC material. Examples of such a selection of areas of perpendicular or parallel orientations for BCP through TC patterning are given in Fig. 4 with UV and e-beam lithography techniques. This intrinsic property leads to processes and integrational paths, which cannot be achieved with classical schemes and materials, and thus further increase the available process-flows, aiming to control and use the selfassembled morphologies.

\subsection{Multilayers stacking}

Another interesting functionality offered by the unique FGH design is tied to their cross-linked and controlled surface energy nature. Indeed, these two features naturally open up the way of an original multilayer stacking process of BCPs films. Previously reported techniques [10] use timeconsuming processes to coat an extra-layer of a material to encapsulate and protect the $\mathrm{BCP}$ film form a potential stripping or lift-off with an upper layer of a second BCP material. In the case of the FGH material, thanks to their spin-coatable nature, the protection of the first BCP layer with its neutral TC is very quickly achieved (few minutes for the cross-linking step) before being able to coat the second layer of BCP. Moreover, the self-assembly bake can be performed either all along or at the end of the stacking process, as the BCP keeps here its own viscoelastic properties along the whole stacking process, conversely to other techniques [11]. Therefore, the overall process is very swift, and a functional two-layer stack can be achieved within less than 10 minutes, as illustrated on the Fig. 5 , left. The stacking process can also include a patterning step, as evidenced in the Fig. 5, right, which can hardly be achieved with other techniques, rendering thus the FGH materials quite appealing for investigations in photonic and metamaterials applications with BCPs.

\section{Conclusion}

We have detailed the design of an innovative TC material combining multiple functionalities. While being able to balance the interfacial tension of a given $\mathrm{BCP}$ material, it demonstrates a high efficiency to suppress the dewetting of the BCP material through the trapping of its initially flat state, without hindering its phase-separation process. The unique patternable ability of this TC design enables new processing schemes for the self-assembly of BCP to select areas of interest in the film and their integration into useful process-flows for nanolithography. Finally, the cross-linked nature opens up interesting potential into photonic/metamaterials applications with BCP, owing to the swift stacking of multiple BCPs layers.

\section{Acknowledgments}

This work was supported by the REX-7 project from Région Rhône Alpes and BPI France, by the French RENATECH network and by the LabEx Minos ANR-10-LABX-55-01. C.G.C., G. H. and G.F. acknowledge financial support from the LCPO/Arkema industrial chair "HOMERIC" (ANR-13-CHIN-0002-01), the Equipex ELORPrintTec ANR-10-EQPX-28-01 with the help of the French state's Initiative d'Excellence IdEx ANR-10-IDEX-003-02. I.I. acknowledges financial support from the Industrial Chair Arkema/CNRSENSAM-Cnam.

\section{References}

1. X. Chevalier, C. Nicolet, R. Tiron, A. Gharbi, M. Argoud, J. Pradelles, M. Delalande, G. Cunge, G. Fleury, G. Hadziioannou, and C. Navarro, Proc. SPIE, 8680 (2013) 868006.

2. C. M. Bates, T. Seshimo, M. J. Maher, W. J. Durand, J. D. Cushen, L. M. Dean, G. Blachut, C. J. Ellison, and C. G. Willson, Science, 338 (2012) 775.

3. X. Chevalier, C. G. Correia, G. Pound-Lana, P. Bézard, M. Sérégé, C. Petit-Etienne, G. Gay, G. Cunge, B. Cabannes-Boué, C. Nicolet, C. Navarro, I. Cayrefourcq, M. Müller, G. Hadziioannou, I. Iliopoulos, G. Fleury, and M. Zelsmann, ACS Appl. Mater. Interfaces, 13 (2021) 11224.

4. X. Chevalier, C. G. Correia, G. Pound-Lana, P. Bézard, M. Sérégé, C. Petit-Etienne, G. Gay, G. Cunge, B. Cabannes-Boué, C. Nicolet, C. Navarro, I. Cayrefourcq, M. Müller, G. Hadziioannou, I. Iliopoulos, G. Fleury, and M. Zelsmann, Proc. SPIE, 11612 (2021) 1161200.

5. A. Legrain, G. Fleury, M. Mumtaz, C. Navarro, J. Arias-Zapata, X. Chevalier, I. Cayrefourcq, M. Zelsmann, ACS Appl. Mater. Interfaces, 9 (2017) 43043.

6. F. Brochard Wyart, P. Martin, and C. Redon, Langmuir, 9 (1993) 3682. 
7. D. P. Sanders, Chem. Rev., 110 (2010) 321.

8. S. Nagahara, C. Q. Dinh, G. Shiraishi, Y. Kamei, K. Nafus, Y. Kondo, M. Carcasi, M. Minekawa, H. Ide, Y. Yoshida, K. Yoshihara, R. Shimada, M. Tomono, K. Takeshita, S. Biesemans, H. Nakashima, D. De Simone, J. S. Petersen, P. Foubert, P. De Bisschop, G. Vandenberghe, H.J. Stock, and B. Meliorisz, Proc. SPIE, 10960, (2019) 109600A.
9. H. Ito, H. "Advances in Polymer Science" Springer, Berlin, Heidelberg, 2005, Vol. 172, p. 37.

10.A. Rahman, P. W. Majewski, G. Doerk, C. T. Black, and K. G. Yager, Nat. Commun. 7 (2016) 13988.

11.H. Jung, D. Hwang, E. Kim, B.-J. Kim, W. B. Lee, J. E. Poelma, J. Kim, C. J. Hawker, J. Huh, D. Y. Ryu, J. Bang, ACS Nano, 5 (2011) 6164. 